\title{
Performance Comparison of SVD- and GMD-assisted MIMO Systems
}

\author{
Andreas Ahrens \\ Wismar University, Germany \\ Email:andreas.ahrens@hs-wismar.de
}

\author{
Cesar Benavente-Peces \\ Universidad Politecnica de Madrid, Spain \\ Email:cesar.benavente@upm.es
}

\author{
Arturs Aboltins \\ Riga Technical University, Latvia \\ Email:aboltins@rtu.lv
}

\begin{abstract}
Singular-value decomposition (SVD)-based multipleinput multiple-output (MIMO) systems have attracted a lot of attention in the wireless community where the whole MIMO channel is decomposed into a number of unequally weighted independent single-input single-output (SISO) channels. The unequal weighting of the SISO channels has led to intensive research on bit- and power allocation even in MIMO channel situations with poor scattering conditions identified as the antenna correlation effect. In this situation, the unequal weighting of the SISO channels becomes even much stronger. In comparison to the SVDassisted MIMO transmission, geometric mean decomposition (GMD)-based MIMO systems are able to compensate for the drawback of weighted SISO channels when using SVD at the cost of remaining interferences which can be easily removed by using dirty paper precoding. Together with different QAM constellation sizes per layer, bit loading and power allocation can be helpful to balance the bit-error probabilities in the activated number of MIMO layers. The novel contribution of this paper is that optimal and suboptimal power allocation solutions are investigated under the assumption of unequal SISO channels as well as different QAM constellation sizes per MIMO layer. Our results show that GMD-based MIMO transmission has the potential to significantly simplify the process of bit and power loading and outperforms the SVD-based MIMO transmission as long as the same QAM-constellation size is used on all equallyweighted SISO channels.
\end{abstract}

Index Terms-Multiple-Input Multiple-Output (MIMO) System; Wireless Transmission; Singular Value Decomposition (SVD); Geometric Mean Decomposition (GMD); Bit Allocation; Power Allocation; Antennas Correlation.

\section{INTRODUCTION}

Current and future communication services demand for higher speed and reliability wireless systems. In order to increase the traditional single-input single-output (SISO) systems transmission capacity additional channels can be added at the cost of increasing the number of transmitter and receiver equipments as well as the overall system cost and complexity. A different and more efficient strategy is exploiting the channel spatial characteristics by spatially distributing multiple antennas at both the transmitter and receiver sides conforming the so-called multiple-input multiple-output (MIMO) system. In a MIMO system different signals are delivered along the various transmitter side antennas at the same time and the same frequency and the multipath signals are simultaneously received at the multiple receiver side antennas and appropriately processed to demodulate the transmitted data. The strategy of placing multiple antennas at the transmitter and receiver sides improves the performance of wireless systems by the use of the spatial characteristics of the channel [1], [2]. Singular-value decomposition (SVD) is well-established in MIMO signal processing where the whole MIMO channel is transferred into a number of independent weighted SISO channels. The unequal weighting of the SISO channels has led to intensive research on bit- and power-allocation in rich and poor scattering conditions [3], [4]. Especially in poor scattering conditions, where the unequal weighting of the SISO channels becomes even stronger, the process of bit- and powerallocation becomes even more challenging. Compared to the SVD-assisted MIMO transmission, geometric mean decomposition (GMD)-based MIMO systems are able to compensate for the drawback of the unequally weighted SISO channels resulting when using SVD [5], [6]. By using the GMD, the whole MIMO system is decomposed into a number of equallyweighted SISO channels at the cost of remaining interferences, which can be easily removed by using dirty paper precoding [7].

However, MIMO channels where antennas are uncorrelated have been largely studied and have reached a state of maturity. In contrast, due to the proximity of the antennas in the transmitter and receiver arrays antennas correlation (also described as spatial correlation) appears, which affects the performance of MIMO systems severely [8], [9], [10]. Thus, antennas-correlated MIMO channels require substantial further research in order to characterize the antennas correlation effect and its influence on the channel performance, which claims for the application of appropriate resource allocation strategies to optimize the MIMO channel performance as described in this work.

The novelty of our contribution is that we demonstrate the benefits of amalgamating a suitable choice of activated MIMO layers and number of bits per layer along with the appropriate allocation of the transmit power under the constraint of a given fixed data throughput. Here, optimal and suboptimal bit- and power-loading in both SVD- and GMD-based MIMO transmission systems are elaborated. Assuming a fixed data rate, which is required in many applications (e.g. real time video applications), a two stage optimization process is proposed. Firstly, the allocation of bits to the number of SISO channels is optimized, and secondly, the allocation of the available total transmit power is studied when minimizing the overall bit- 
error rate (BER). Whereas optimal power allocation techniques are highly complex, the proposed suboptimal solutions based on the equal signal-to-noise ratio (SNR) condition offer a good compromise between complexity and performance loss compared with optimal solutions.

Our results show that GMD-based MIMO transmission has the potential to significantly simplify the process of bit and power loading both in correlated and uncorrelated MIMO systems and outperforms SVD-based MIMO transmission as long as the same QAM-constellation size is used on all equalweighted SISO channels.

The remaining part of this paper is structured as follows: Section II describes the MIMO system model and the corresponding signal processing techniques. The quality criteria used in this work are shortly reviewed in section III. The investigated resource allocation techniques such as bit- and power allocation are discussed in section IV. The obtained results are presented and interpreted in section V. Finally section VI remarks the main conclusions obtained in this investigation.

\section{MIMO SYSTEM MODEL}

When considering a non-frequency selective SDM (space division multiplexing) MIMO link composed of $n_{\mathrm{T}}$ transmit and $n_{\mathrm{R}}$ receive antennas, the system is modelled by

$$
\mathbf{u}=\mathbf{H} \cdot \mathbf{c}+\mathbf{n} .
$$

In (1), $\mathbf{u}$ is the $\left(n_{\mathrm{R}} \times 1\right)$ received data vector, $\mathbf{c}$ is the $\left(n_{\mathrm{T}} \times 1\right)$ transmitted signal vector containing the complex input symbols and $\mathbf{n}$ is the $\left(n_{\mathrm{R}} \times 1\right)$ vector of the additive, white Gaussian noise (AWGN) having a variance of $U_{\mathrm{R}}^{2}$ for both the real and imaginary parts.

Furthermore, it is assumed that the coefficients of the channel matrix $\mathbf{H}$ are independently Rayleigh distributed with equal variance and that the number of transmit antennas $n_{\mathrm{T}}$ equals the number of receive antennas $n_{\mathrm{R}}$.

However, due to the proximity of the antennas at the transmitter as well as the receiver side, the assumption of independently Rayleigh distributed channel elements is not any longer valid [11]. The channel matrix of a correlated MIMO system can be expressed as

$$
\operatorname{vec}(\mathbf{H})=\mathbf{R}_{\mathrm{HH}}^{1 / 2} \cdot \operatorname{vec}(\mathbf{G}),
$$

where $\mathbf{G}$ is the $\left(n_{\mathrm{R}} \times n_{\mathrm{T}}\right)$ uncorrelated channel matrix with independent, identically distributed complex valued Rayleigh elements and vec $(\cdot)$ is the operator stacking a given matrix into a vector column-wise. The expression $(\cdot)^{1 / 2}$ defines the matrix square-root operator. Following the quite common assumption that the correlation between the antenna elements at the transmitter side (described by the transmitter side correlation matrix $\mathbf{R}_{\mathrm{TX}}$ ) is independent from the correlation between the antenna elements at the receiver side (described by the receiver side correlation matrix $\mathbf{R}_{\mathrm{RX}}$ ), the correlation matrix $\mathbf{R}_{\mathrm{HH}}$ can be described by

$$
\mathbf{R}_{\mathrm{HH}}=\mathbf{R}_{\mathrm{TX}} \otimes \mathbf{R}_{\mathrm{RX}}
$$

where $\otimes$ states the Kronecker product.

A popular technique to process the MIMO channel is based on the singular value decomposition (SVD) [12] of the system matrix $\mathbf{H}$, which can be written as $\mathbf{H}=\mathbf{S} \cdot \mathbf{V} \cdot \mathbf{D}^{\mathrm{H}}$, where $\mathbf{S}$ and $\mathbf{D}^{\mathrm{H}}$ are unitary matrices and $\mathbf{V}$ is a real-valued diagonal matrix of the positive square roots of the eigenvalues of the matrix $\mathbf{H}^{\mathrm{H}} \mathbf{H}$ sorted in descending order. The conjugate transpose (Hermitian) of $\mathbf{D}$ is denoted by $\mathbf{D}^{\mathrm{H}}$. Using the SVD, the whole MIMO system is transformed into independent, noninterfering layers having unequal gains. The resulting SVDbased layer-specific MIMO system model is highlighted in Fig. 1. Here, the layer-specific data symbol at the time $k$ i. e., $c_{\ell, k}$, is multiplied by the weighting factor $\sqrt{\xi_{\ell, k}}$ (for $\ell=1,2, \ldots, L$ and $L$ defining the number of activated MIMO layers), as the positive square roots of the eigenvalues of the matrix $\mathbf{H}^{\mathrm{H}} \mathbf{H}$, and disturbed by the additive white Gaussian component $w_{\ell, k}$ resulting in the layer-specific receive data symbol $y_{\ell, k}$.

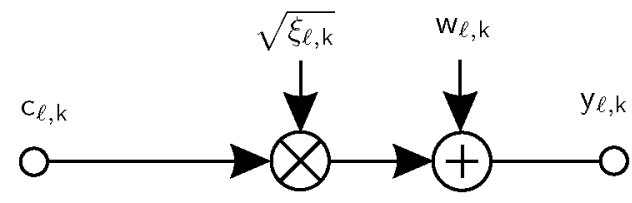

Fig. 1. System model per MIMO layer $\ell$ and transmitted data block $k$ after SVD pre- and post-processing

To analyse the correlation effect, the ratio $\vartheta$ between the smallest and the largest singular values seems to be an unique indicator of the unequal weighting of the MIMO layers. Fig. 2 shows the probability density function (PDF) of the $\vartheta$ for uncorrelated and correlated frequency non-selective $(4 \times 4)$ MIMO systems. Here it is worth mentioning that the number of easily separable MIMO layers is limited by the minimum numbers of antennas at both sides, the transmitter as well as the receiver side. Fig. 2 illustrates how the ratio between the singular values increases (i.e the unequal weighting) as the correlation does. This means that the ratio between the smallest and the largest singular value decreases, and then, the probability of having predominant layers increases. As a result, the use of resource allocation techniques seems an appropriate solution to optimize the layer behaviour since no power should be allocated to the MIMO layer having the smallest singular values because of the overall performance would be deteriorated.

On the other hand, GMD decomposes the channel matrix into

$$
\mathbf{H}=\mathbf{Q} \cdot \mathbf{R} \cdot \mathbf{P}^{\mathrm{H}},
$$

where the $\left(n_{\mathrm{R}} \times n_{\mathrm{R}}\right)$ matrix $\mathbf{Q}$ and the $\left(n_{\mathrm{T}} \times n_{\mathrm{T}}\right)$ matrix $\mathbf{P}$ are composed of orthogonal columns, and $\mathbf{R}$ is a real-valued upper triangular matrix where the off-diagonal elements represent the remaining interferences and all the elements in the main diagonal take the same value which is the geometric mean of the positive square roots of the eigenvalues of the matrix $\mathbf{H}^{\mathrm{H}} \mathbf{H}$ given by (see also Fig. 3). 


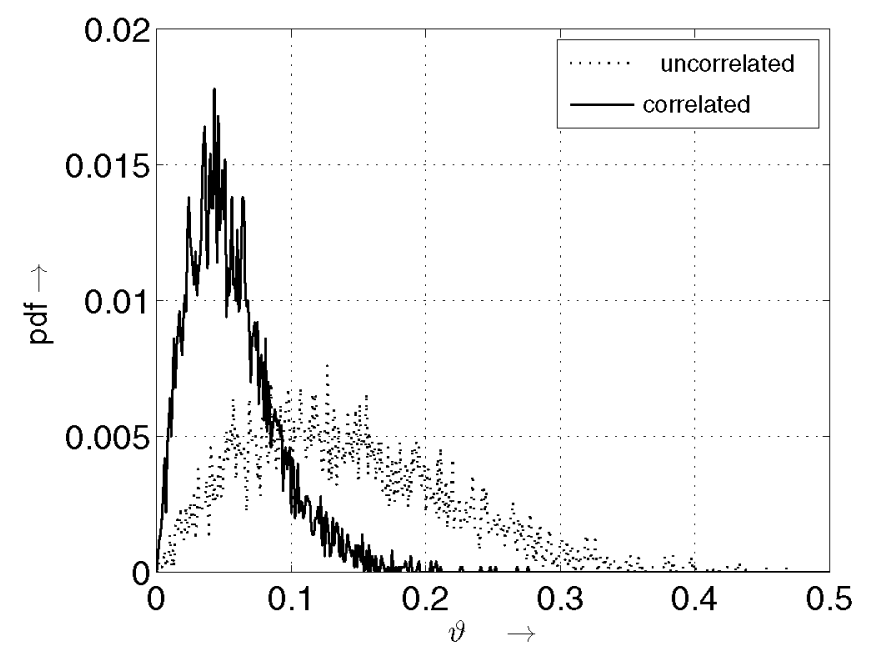

Fig. 2. PDF of the ratio $\vartheta$ between the smallest and the largest singular value for uncorrelated (dotted line) as well as correlated (solid line) frequency nonselective $(4 \times 4)$ MIMO channel

$$
\sqrt{\xi_{k}}=\left(\prod_{\ell=1}^{L} \sqrt{\xi_{\ell, k}}\right)^{(1 / L)}
$$

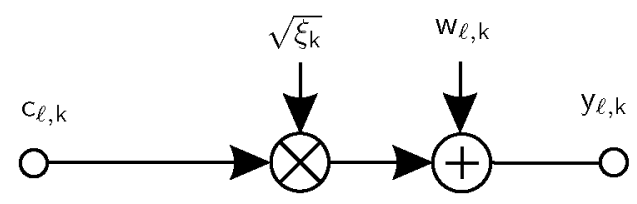

Fig. 3. System model per MIMO layer $\ell$ and transmitted data block $k$ after GMD pre- and post-processing

Once the channel matrix $\mathbf{H}$ is described in terms of the GMD given in (4) the MIMO system requires appropriate preand post-processing in order to transform the MIMO system into multiple SISO channels and the transmission system results in

$$
\mathbf{y}=\mathbf{Q}^{\mathrm{H}} \cdot \mathbf{u}=\mathbf{Q}^{\mathrm{H}}(\mathbf{H} \cdot \mathbf{P} \cdot \mathbf{c}+\mathbf{n}),
$$

and can be represented as

$$
\mathbf{y}=\mathbf{R} \cdot \mathbf{c}+\mathbf{w}
$$

where $\mathbf{y}$ is the $\left(n_{\mathrm{R}} \times 1\right)$ post-processed data vector at the receiver side and $\mathbf{w}=\mathbf{Q}^{\mathrm{H}} \cdot \mathbf{n}$ is the $\left(n_{\mathrm{R}} \times 1\right)$ post-processed noise vector.

By using perfect interference cancellation (e.g. TomlinsonHarshima precoding) the remaining interferences can be removed perfectly resulting in a system model that can be described as

$$
\mathbf{y}=\tilde{\mathbf{R}} \cdot \mathbf{c}+\mathbf{w}
$$

where $\tilde{\mathbf{R}}$ is a $\left(n_{\mathrm{R}} \times n_{\mathrm{T}}\right)$ diagonal matrix whose non-zero elements equal the geometric mean of the singular values of $\mathbf{H}^{\mathrm{H}} \mathbf{H}$. In this work the GMD-based preprocessing for removing the remaining system-specific interferences are not analysed. It is assumed that these interferences can be completely eliminated. The required signal processing in both SVD- and GMD-based MIMO transmission systems modifies neither the transmit power nor the noise levels since the preand post-processing matrices are unitary.

Fig. 4 compares for an exemplary frequency non-selective $(4 \times 4)$ MIMO system the singular values in the SVD-based system matrix $\mathbf{V}$ (a) with the GMD-based system matrix $\mathbf{R}$ (b) where interferences still remain. Furthermore, the matrix $\tilde{\mathbf{R}}$ is shown for 4 active layers (c), 3 active layers (d), 2 active layers (e) and 1 active layer (f). It is worth noting that based on equation (5) the geometric mean increases as a lower number of active layers are considered. This is due to the existence of weak layers with low gain coefficients, which can be clearly identified in Fig. 4 (a).

\section{QUALITY CRITERIA}

The quality criteria considered for the end-to-end wireless communication system performance is given in terms of the bit-error-rate (BER), which quantifies the reliability of the entire wireless system from input to output.

In order to optimize the overall channel performance, as shown below, the argument of the complementary error function, also known as signal-to-noise ratio (SNR), is maximized as an alternative to minimize the BER. The SNR per quadrature component can be defined as

$$
\varrho=\frac{(\text { Half vertical eye opening })^{2}}{\text { Noise Power }}=\frac{\left(U_{\mathrm{A}}\right)^{2}}{\left(U_{\mathrm{R}}\right)^{2}},
$$

where $U_{\mathrm{A}}$ is the half vertical eye opening and $U_{\mathrm{R}}^{2}$ is the noise power per quadrature component taken at the detector input. The relationship between the signal-to-noise ratio $\varrho$ and the bit-error probability evaluated for AWGN channels and M-ary Quadrature Amplitude Modulation (QAM) is given by

$$
P_{\mathrm{b}}=\frac{2}{\log _{2}(M)} \cdot\left(1-\frac{1}{\sqrt{M}}\right) \cdot \operatorname{erfc}\left(\sqrt{\frac{\varrho}{2}}\right) .
$$

The application of the SVD pre- and post-processing leads to an unequally weighted SISO channels (as illustrated in Fig. 1) with different eye openings per activated MIMO layer $\ell$ and per transmitted symbol block $k$ according to

$$
U_{\mathrm{A}}^{(\ell, k)}=\sqrt{\xi_{\ell, k}} \cdot U_{\mathrm{s} \ell} .
$$

Considering QAM constellations, the average transmit power per MIMO layer $P_{\mathrm{s} \ell}$ may be expressed as a function of the half-level transmit amplitude $U_{\mathrm{s} \ell}$ and the layer-specific constellation size $M_{\ell}$ as

$$
P_{\mathrm{s} \ell}=\frac{2}{3} U_{\mathrm{s} \ell}^{2}\left(M_{\ell}-1\right) .
$$

By taking $L \leq \min \left(n_{\mathrm{T}}, n_{\mathrm{R}}\right)$ MIMO activated layers into account, the overall transmit power results in 
(a)

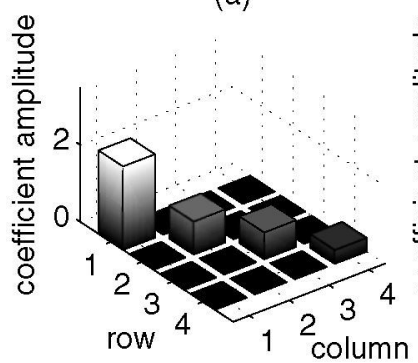

(d)

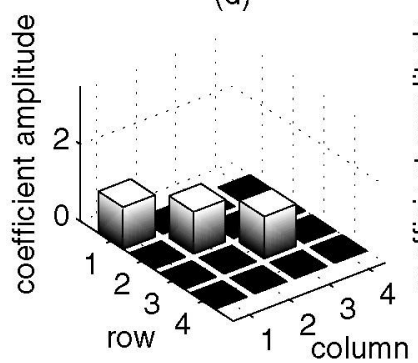

(b)

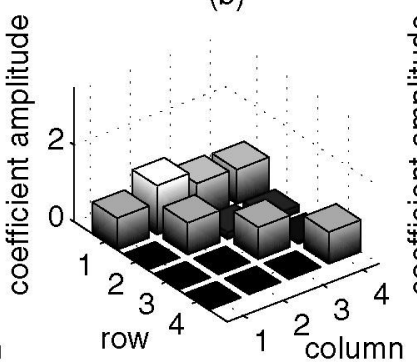

(e)

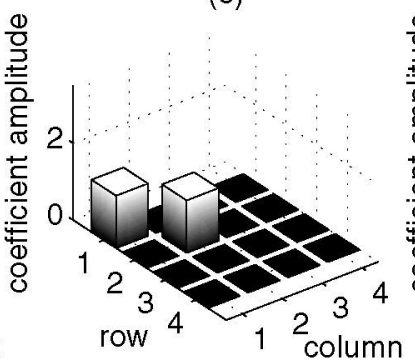

(c)

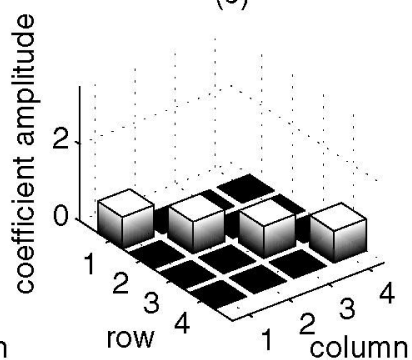

(f)

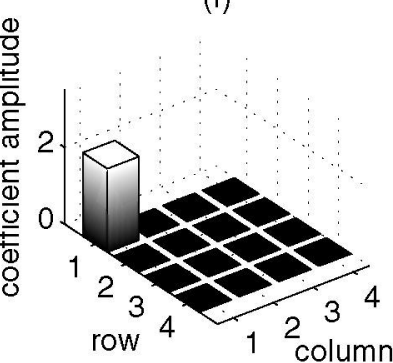

Fig. 4. Singular values and geometric mean comparison (a) matrix $\mathbf{V}, \mathbf{b}$ ) matrix $\mathbf{R}, \mathrm{c}, \mathrm{d}, \mathbf{r}, \mathbf{f}$ ) matrix $\tilde{\mathbf{R}}$ matrix for a different number of activated layers).

$$
P_{\mathrm{s}}=\sum_{\ell=1}^{L} P_{\mathrm{s} \ell}
$$

where $P_{\mathrm{S}}$ is the total available power at the transmit side. Finally, the layer-specific bit-error probability at the time slot $k$ is obtained by combining (9), (10), and (11) resulting in

$$
P_{\mathrm{b}}^{(\ell, k)}=\frac{2}{\log _{2}\left(M_{\ell}\right)}\left(1-\frac{1}{\sqrt{M_{\ell}}}\right) \operatorname{erfc}\left(\frac{U_{\mathrm{A}}^{(\ell, k)}}{\sqrt{2} U_{\mathrm{R}}}\right)
$$

The aggregate bit-error probability at the time slot $k$, taking $L$ activated MIMO-layers into account, results in

$$
P_{\mathrm{b}}^{(k)}=\frac{1}{\sum_{\nu=1}^{L} \log _{2}\left(M_{\nu}\right)} \sum_{\ell=1}^{L} \log _{2}\left(M_{\ell}\right) P_{\mathrm{b}}^{(\ell, k)} .
$$

Finally, the BER of the whole MIMO system is obtained by considering the different transmission block SNRs. In order to balance the bit error probability along the MIMO system activated layers, bit and power loading provides helpful strategies to improve the overall performance. The bit error probability at a given time $k$ is influenced by both the chosen QAM constellation and the layer-specific weighting factors. In particular, the layer-specific weighting factors influence the overall performance.

When using GMD with different QAM constellation sizes per MIMO layer instead of the SVD pre- and post-processing, unequally weighted layers (as illustrated in Fig. 3) appear according to

$$
U_{\mathrm{A}}^{(\ell, k)}=\sqrt{\xi_{k}} \cdot U_{\mathrm{s} \ell},
$$

resulting in different qualities per activated MIMO layer. However when transmitting with the same QAM constellation size on each MIMO input, equally weighted MIMO layers appear. Together with an identical noise power at the detector's input, the same quality is guaranteed on all activated MIMO layers.

\section{RESSOURCE Allocation In SVD-BASEd MiMO SYSTEMS}

Resource allocation strategies allow the optimization of the MIMO channel overall performance. Hence, the BER can be minimized under the constraints of a fixed data rate and a limited available transmit power. Regarding the channel quality, the BER performance is affected by both the layerspecific weighting factors $\sqrt{\xi_{\ell, k}}$ and the QAM-constellation size $M_{\ell}$. Assuming a fixed data rate, regardless of the channel quality, Table I highlights the resulting layer-specific QAM constellations for a fixed spectral efficiency of $8 \mathrm{bit} / \mathrm{s} / \mathrm{Hz}$.

Following the allocation of bits per layer, power allocation (PA) can be added to optimize the overall BER. The layerspecific power allocation weights $\sqrt{p_{\ell, k}}$ adjust the half-vertical eye opening per symbol block as follows (see Fig. 5)

$$
U_{\mathrm{APA}}^{(\ell, k)}=\sqrt{p_{\ell, k}} \cdot \sqrt{\xi_{\ell, k}} \cdot U_{\mathrm{s} \ell} .
$$

This results in the layer-specific transmit power per symbol 
TABLE I

Investigated QAM transmission modes assuming $n_{\mathrm{R}}=n_{\mathrm{T}}=4$

\begin{tabular}{ccccc}
\hline throughput & layer 1 & layer 2 & layer 3 & layer 4 \\
\hline $8 \mathrm{bit} / \mathrm{s} / \mathrm{Hz}$ & 256 & 0 & 0 & 0 \\
$8 \mathrm{bit} / \mathrm{s} / \mathrm{Hz}$ & 64 & 4 & 0 & 0 \\
$8 \mathrm{bit} / \mathrm{s} / \mathrm{Hz}$ & 16 & 16 & 0 & 0 \\
$8 \mathrm{bit} / \mathrm{s} / \mathrm{Hz}$ & 16 & 4 & 4 & 0 \\
$8 \mathrm{bit} / \mathrm{s} / \mathrm{Hz}$ & 4 & 4 & 4 & 4 \\
\hline
\end{tabular}

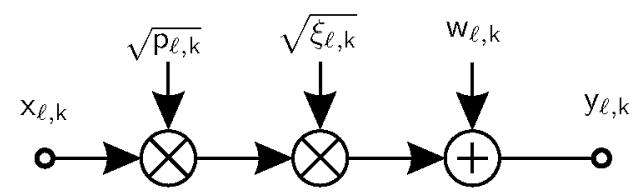

Fig. 5. Resulting layer-specific system model including MIMO-layer PA

block $k$

$$
P_{\mathrm{s} P A}^{(\ell, k)}=p_{\ell, k} \cdot P_{\mathrm{s} \ell}
$$

where $P_{\mathrm{s} \ell}$ denotes the allocated power per MIMO layer without PA e.g. $P_{\mathrm{s} \ell}=P_{\mathrm{s}} / L$. Therein the parameter $L$ describes the number of activated MIMO layers. Taking all activated MIMO layers $L$ into account, being $L \leq \min \left(n_{\mathrm{T}}, n_{\mathrm{R}}\right)$, the overall transmit power per symbol block $k$ is obtained as

$$
P_{\mathrm{sPA}}^{(k)}=\sum_{\ell=1}^{L} P_{\mathrm{sPA}}^{(\ell, k)} .
$$

With (17) the layer-specific bit-error probability at the time $k$ becomes

$$
P_{\mathrm{bPA}}^{(\ell, k)}=\frac{2}{\log _{2}\left(M_{\ell}\right)}\left(1-\frac{1}{\sqrt{M_{\ell}}}\right) \operatorname{erfc}\left(\frac{U_{\mathrm{APA}}^{(\ell, k)}}{\sqrt{2} U_{\mathrm{R}}}\right) .
$$

In order to find the optimal set of PA parameters minimizing the overall BER, i.e., $\sqrt{p_{\ell, k}}$, the Lagrange multiplier method is used. The cost function for this method $J\left(p_{1, k}, p_{2, k}, \ldots, p_{L, k}\right)$ may be expressed as

$$
J(\cdots)=\frac{1}{\sum_{\nu=1}^{L} \log _{2}\left(M_{\nu}\right)} \sum_{\ell=1}^{L} \log _{2}\left(M_{\ell}\right) P_{\mathrm{b}}^{(\ell, k)}+\lambda \cdot B
$$

where $\lambda$ denotes the Lagrange multiplier. The parameter $B$ in (21) describes the boundary condition to meet the overall transmit power constraints

$$
\begin{aligned}
B & =\sum_{\ell=1}^{L}\left(P_{\mathrm{s} \ell}-P_{\mathrm{sPA}}^{(\ell, k)}\right)=0 \\
& =\sum_{\ell=1}^{L} P_{\mathrm{s} \ell}\left(1-p_{\ell, k}\right)=0 .
\end{aligned}
$$

TABLE II

Investigated channel profiles for studying the effect of optimum power allocation

\begin{tabular}{ccccc}
\hline Profile & layer 1 & layer 2 & layer 3 & layer 4 \\
\hline $\mathrm{CM}-1$ & 1,751 & 0,871 & 0,436 & 0,214 \\
$\mathrm{CM}-2$ & 1,903 & 0,624 & 0,212 & 0,0692 \\
\hline
\end{tabular}

Together with the natural choice of distributing the available transmit power uniformly over the number of activated MIMO layers, i. e., $P_{\mathrm{s} \ell}=P_{\mathrm{s}} / L$, the boundary condition results in

$$
B=\frac{P_{\mathrm{s}}}{L} \sum_{\ell=1}^{L}\left(1-p_{\ell, k}\right)=0
$$

where the transmit power coefficients have to fulfill the condition

$$
\sum_{\ell=1}^{L} p_{\ell, k}=L
$$

Differentiating the Lagrangian cost function $J\left(p_{1, k}, p_{2, k}, \ldots, p_{L, k}\right)$ with respect to the $p_{\ell, k}$ and setting it to zero, leads to the optimal set of PA parameters. In order to analyse the effect of PA thoroughly, the fixed channel profiles shown in Table II are investigated. For comparison reasons, the channel profile CM-1 describes a MIMO channel with a low degree of correlation $(\vartheta=0,122)$, defined by the ratio of the lowest singular value and the largest singular value, whereas the channel CM-2 introduces a higher degree of antennas' correlation ( $\vartheta=0,036$ ). In this case the unequal weighting of the layers becomes stronger compared to the channel profile CM-1.

Since the optimal PA solution is notably computationally complex to implement, a suboptimal solution which concentrates on the argument of the complementary error function is investigated. In this particular case the signal-to-noise ratio

$$
\varrho_{\mathrm{PA}}^{(\ell, k)}=\frac{\left(U_{\mathrm{A} P \mathrm{PA}}^{(\ell, k)}\right)^{2}}{U_{\mathrm{R}}^{2}}
$$

is set to be equal for all activated MIMO layers per data block $k$, i. e., $\varrho_{\mathrm{PA}}^{(\ell, k)}=\mathrm{constant} \quad \ell=1,2, \ldots, L$.

Assuming that initially the transmit available power is uniformly distributed along the active layers, the power allocation coefficient for each MIMO layer $\ell$ and transmitted data block $k$ can be determined by

$$
p_{\ell, k}=\frac{\left(M_{\ell}-1\right)}{\xi_{\ell, k}} \cdot \frac{L}{\sum_{\nu=1}^{L} \frac{\left(M_{\nu}-1\right)}{\xi_{\nu, k}}} .
$$

Hence, for each symbol the same half vertical eye opening of (17) can be guaranteed $(\ell=1, \ldots, L)$, i.e.,

$$
U_{\mathrm{A} P A}^{(\ell, k)}=\text { constant } \quad \ell=1,2, \ldots, L .
$$

Considering an identical noise power at the detector's input, the above-mentioned equal quality scenario is encountered. 
TABLE III

Computational load of the investigated PA methods assuming a $(4 \times 4)$ MIMO system at $10 \log _{10}\left(E_{\mathrm{s}} / N_{0}\right)=20 \mathrm{~dB}$

\begin{tabular}{ccc}
\hline Power Allocation & Memory & Time \\
\hline Optimal & $9.80 \mathrm{MiB}$ & $200.00 \mathrm{~ms}$ \\
Suboptimal & $0.22 \mathrm{MiB}$ & $5.00 \mathrm{~ms}$ \\
\hline
\end{tabular}

\section{REsUlts}

In this work the efficiency of resource allocation strategies is investigated under the constraints of a fixed data rate and a limited available transmit power, where the obtained BER performance is affected by both the SVD-based layerspecific weighting factors $\sqrt{\xi_{\ell, k}}$ or the GMD-based layerspecific weighting factors $\sqrt{\xi_{k}}$ and the QAM-constellation sizes $M_{\ell}$ expressed by different layer-specific weighting factors described in section II. Assuming a fixed data rate, regardless of the channel quality, Table I highlights the resulting layer-specific QAM constellations for a fixed data throughput considering a different number of activated layers and bits per activated layer.

In order to highlight the effect of resource allocation strategies, fixed exemplary channel profiles such as CM-1 and CM2 are defined (see Table II). The SVD-based BER curves for channel profiles CM-1 and CM-2 are shown in Fig. 6 and Fig. 7 when using optimal power allocation together with the beforehand defined fixed transmission modes. Here it can be seen that when using the MIMO channel in an optimized way not all the MIMO layers should be necessarily activated to obtain the best performance.

In Fig. 8 the obtained BER curves with the optimal PA based on the Lagrange multiplier method are compared with the above mentioned equal quality criteria. As demonstrated by computer simulations the loss in the overall BER with the equal quality criteria is quite acceptable when using an optimized bit loading, i. e., the performance loss is low while the computational load is largely reduced.

Table III compares the memory usage and the corresponding processing time taken to execute the optimal and suboptimal power solutions with an AMD A4-5300 APU processor with a clock of $3.40 \mathrm{GHz}$. It turned out that the proposed suboptimal equal-SNR PA technique requires a lower computational load compared with the optimal one. As shown by our simulation, the suboptimum solution can be calculated with a computational load about 40 times lower compared to the optimal one.

In order to analyse the advantages of equally-weighted channels, the previously presented results have to be adopted to the GMD-based ones. The investigated channel profiles highlighted in Table IV and V, are obtained from the channel profiles $\mathrm{CM}-1$ and $\mathrm{CM}-2$ when calculating the geometric mean of the corresponding singular values for the different number of activated layers.

Fig. 9 depicts and compares the performance of the GMDbased MIMO systems for the transmission modes shown in

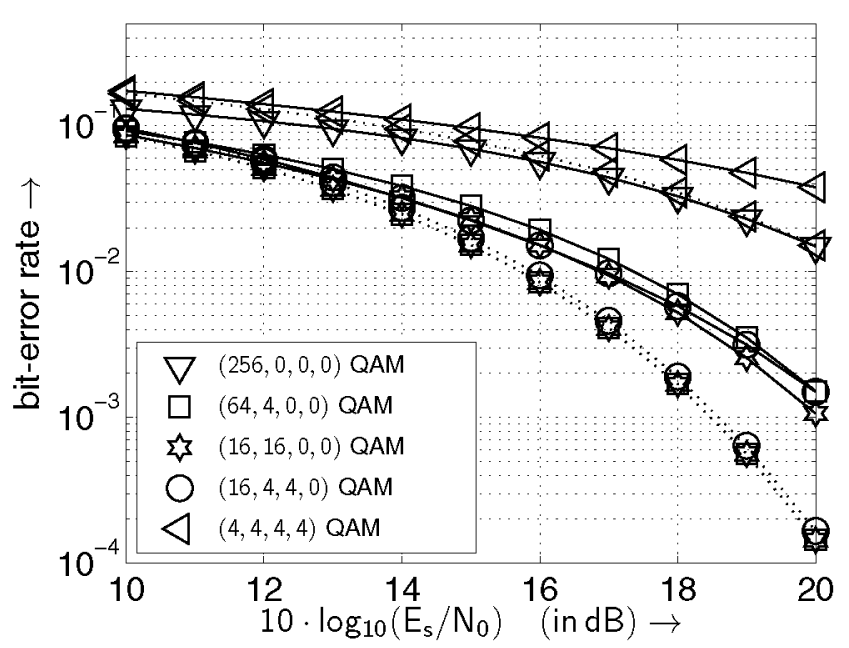

Fig. 6. SVD-based BER curves with optimal PA (dotted line) and without PA (solid line) when using the transmission modes introduced in Table I and transmitting $8 \mathrm{bit} / \mathrm{s} / \mathrm{Hz}$ over channel $\mathrm{CM}-1$

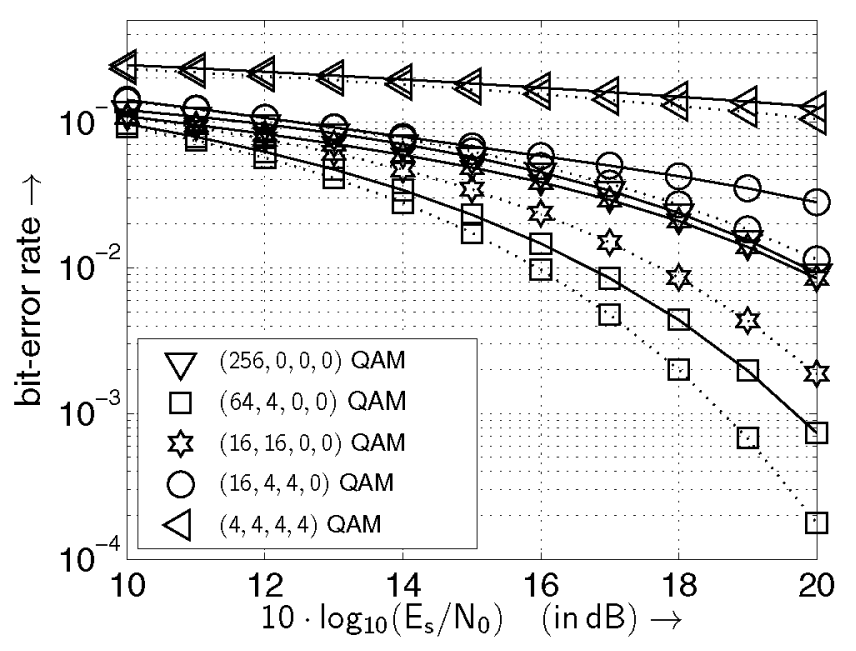

Fig. 7. SVD-based BER curves with optimal PA (dotted line) and without PA (solid line) when using the transmission modes introduced in Table I and transmitting $8 \mathrm{bit} / \mathrm{s} / \mathrm{Hz}$ over channel $\mathrm{CM}-2$

Table I and the channel profile CM-3.x defined in Table IV, where ' $x$ ' states for the number of activated layers. In channel situation where the transmitted bits are uniformly distributed over the number of activated MIMO layers, power allocation has no effect and can be excluded in order to save some computational load. In general terms the best performance is obtained when the channel energy, represented by the layerspecific weighting factors, is concentrated on a certain number of MIMO layers. Taking all layers as well as only one layer into account, the best performance is not necessarily guaranteed. As shown in Fig. 9 the best performance is obtained for transmission mode $(16,16,0,0)$. In those transmission modes where the bits are not uniformly distributed over the number of activated MIMO-layers the benefits of using power allocation 


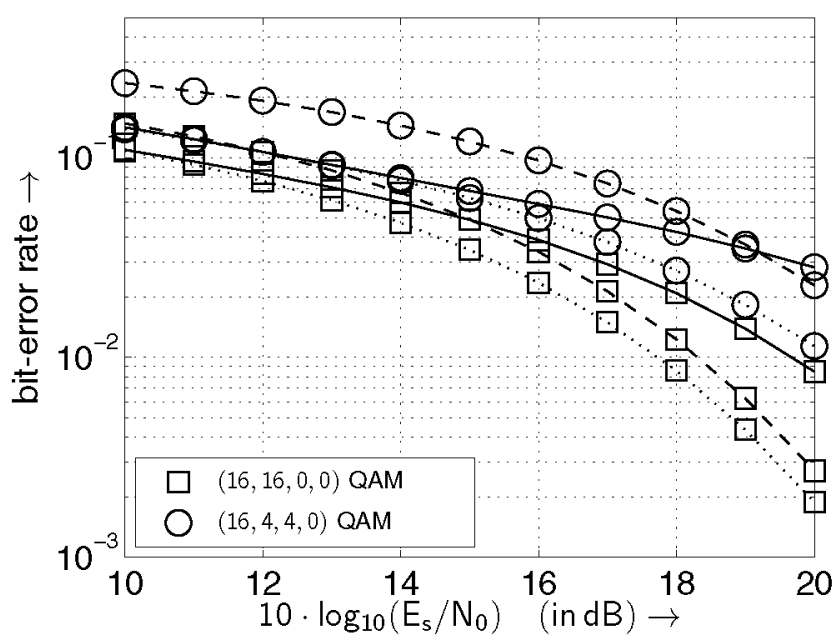

Fig. 8. SVD-based BER curves with optimal PA (dotted line), equal-SNR PA (dashed line) and without PA (solid line) when using the best performance transmission modes introduced in Table I and transmitting $8 \mathrm{bit} / \mathrm{s} / \mathrm{Hz}$ over channel CM-2

TABLE IV

Investigated GMD-based channel profiles using channel CM-1

\begin{tabular}{ccccc}
\hline Profile & layer 1 & layer 2 & layer 3 & layer 4 \\
\hline $\mathrm{CM}-3.1$ & 1,751 & -- & -- & -- \\
$\mathrm{CM}-3.2$ & 1,235 & 1,235 & -- & -- \\
$\mathrm{CM}-3.3$ & 0,873 & 0,873 & 0,873 & -- \\
$\mathrm{CM}-3.4$ & 0,614 & 0,614 & 0,614 & 0,614 \\
\hline
\end{tabular}

are clearly visible.

It is worth noting that with increasing the number of active layers, the layer specific gain coefficient decreases since the calculation of the geometric mean includes low-valued singular values.

Fig. 10 shows the performance of a GMD-based MIMO system for the transmission modes CM-4.x described in Table $\mathrm{V}$. This channel is obtained from the channel profile CM2 , where a strong unequal weighting of the SVD-based layer specific weighting factors is assumed. Based on the stronger unequal weighting, the corresponding geometric mean values become lower compared with the channel CM-3.x, where the SVD-based layer specific weighting factors are more equally distributed. Except for the activation of only one layer, the GMD performance becomes lower compared with the channel CM-3.x. However, the increase in the layer-specific weighting factor when activating only one layer does not lead to the best performance. Here, the $(16,16,0,0)$ transmission mode shows again the best performance.

Fig. 11 compares the performances of the SVD-based MIMO system versus the GMD-based one for the transmission mode $(16,16,0,0)$. As long as the bits are uniformly distributed over the number of activated MIMO layers, the proposed GMD-based signal processing, which does not require any power allocation, shows a lower BER compared
TABLE V

Investigated GMD-based channel profiles using channel CM-2

\begin{tabular}{ccccc}
\hline Profile & layer 1 & layer 2 & layer 3 & layer 4 \\
\hline CM-4.1 & 1,903 & -- & -- & -- \\
CM-4.2 & 1,090 & 1,090 & -- & -- \\
CM-4.3 & 0,631 & 0,631 & 0,631 & -- \\
CM-4.4 & 0,363 & 0,363 & 0,363 & 0,363 \\
\hline
\end{tabular}

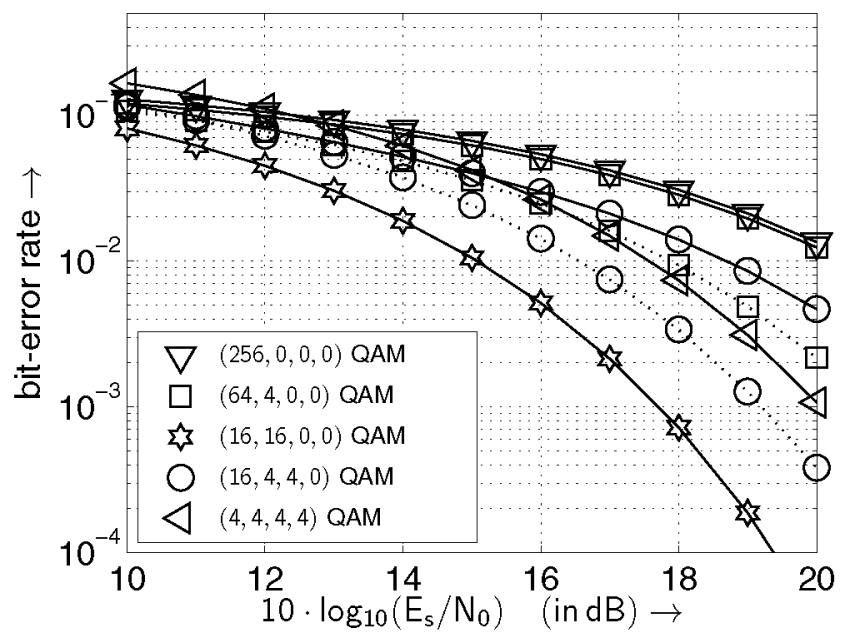

Fig. 9. GMD-based BER curves with optimal PA (dotted line) and without PA (solid line) when using the transmission modes introduced in Table I and transmitting $8 \mathrm{bit} / \mathrm{s} / \mathrm{Hz}$ over channel $\mathrm{CM}-3 . x$

with the optimised SVD-based solution.

\section{CONClusion}

In this work both SVD- and GMD-assisted MIMO systems have been investigated in channel situations with and without spatial correlation. In order to achieve the best BER performance a two-stage optimization approach has been presented. Firstly, the bits are allocated to the activated MIMO layers and secondly, different power allocation strategies have been studied in order to get the best BER performance. As demonstrated in this work, it turned out that the choice of the number of bits per symbol as well as the number of activated MIMO layers substantially affects the performance of a MIMO system especially in situations with strongly unequal weighted layers also referred to spatial correlation, suggesting that not all MIMO layers have to be activated in order to achieve the best BER performance.

This investigation has demonstrated that GMD-based signal processing shows the advantage of a lower BER and a lower computational load compared with SVD-based solutions as long as the number of transmitted bits is uniformly distributed over the activated MIMO layers. Then, GMD-based MIMO systems constitute a promising alternative to SVD-based ones in future communication systems. 


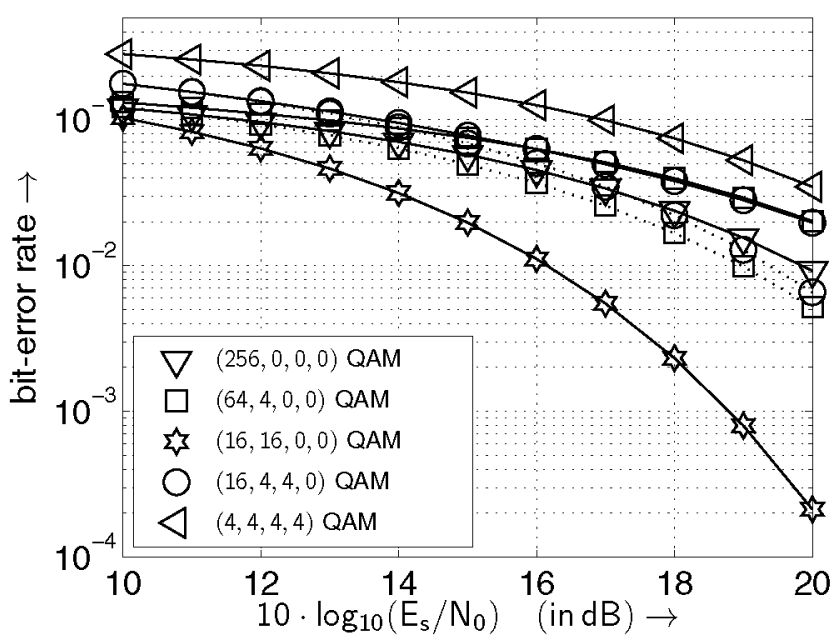

Fig. 10. GMD-based BER curves with optimal PA (dotted line) and without PA (solid line) when using the transmission modes introduced in Table I and transmitting $8 \mathrm{bit} / \mathrm{s} / \mathrm{Hz}$ over channel $\mathrm{CM}-4 . x$

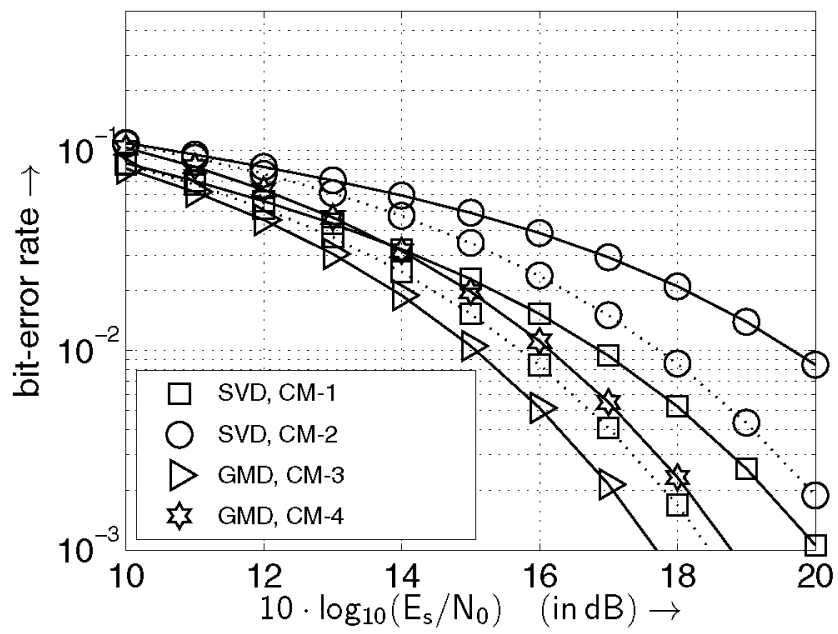

Fig. 11. Comparison of SVD- and GMD-based BER curves with optimal PA (dotted line) and without PA (solid line) when using the $(16,16,0,0)$ transmission modes introduced in Table I and transmitting $8 \mathrm{bit} / \mathrm{s} / \mathrm{Hz}$ over channel CM-1 - CM-4

\section{REFERENCES}

[1] P. Yang, Y. Xiao, Y. Yu, and S. Li, "Adaptive Spatial Modulation for Wireless MIMO Transmission Systems," IEEE Communications Letters, vol. 15 , pp. 602-604, 2011.

[2] M. Chiani, M. Win, and A. Zanella, "On the Capacity of Spatially Correlated MIMO Rayleigh-fading Channels," IEEE Transactions on Information Theory, vol. 49, pp. 2363-2371, 2003.

[3] F. Cano-Broncano, A. Ahrens, and C. Benavente-Peces, "Iterative Bitand Power Allocation in Correlated MIMO Systems," in Intemational Conference on Pervasive and Embedded Computing and Communication Systems (PECCS), Lisboa, (Portugal), 7-9 January 2014.

[4] H. Chen and Y. Lin, "Limited Feedback of Precoder and Bit Loading for MIMO Systems: A Joint Design," IEEE Transactions on Signal Processing, vol. 61, no. 23, pp. 6091-6102, 2013.

[5] Y. Jiang, J. Li, and W. Hager, "Joint Transceiver Design for MIMO Communications Using Geometric Mean Decomposition," IEEE Transactions on Signal Processing, vol. 53, pp. 3791-3803, 2005.
[6] Y. Jiang, W. W. Hager, and J. Li, "The Geometric Mean Decomposition." Linear Algebra and Its Applications, pp. 373-384, 2005.

[7] C.-C. Weng, C.-Y. Chen, and P. Vaidyanathan, "MIMO Tranceivers with Decision Feedback and Bit Loading: Theory and Optimization," IEEE Transactions on Signal Processing, vol. 58, pp. 1334-1346, 2010.

[8] S. Loyka and G. Tsoulos, "Estimating MIMO System Performance using the Correlation Matrix Approach," IEEE Communications Letters, vol. 6, pp. $19-21,2002$.

[9] D.-S. Shiu, F. G.J, M. Gans, and J. Kahn, "Fading Correlation and its effect on the Capacity of multi-element antenna systems," in Universal Personal Communications, 1998

[10] H. Wang, P. Wang, L. Ping, and X. Lin, "How does Correlation Affect the Capacity of MIMO Systems with Rate Constraints?" in Global Telecommunications Conference, 2009.

[11] A. Ahrens, F. Cano-Broncano, and C. Benavente-Peces, "Power Distribution for SVD-aided MIMO Transmission with Receiver-Side Antennas Correlation," in 10th IASTED International Conference on Signal Processing, Pattern Recognition, and Applications (SPPRA) Innsbruck (Austria), 2013, pp. $12-14$.

[12] S. S. Haykin, Adaptive Filter Theory. New Jersey: Prentice Hall, 2002. 